1965

\title{
The Intentionality Structure of Complementarity
}

Patrick A. Heelan

Georgetown University, heelanp@georgetown.edu

Follow this and additional works at: https://fordham.bepress.com/phil_research

Part of the Continental Philosophy Commons, Philosophy of Science Commons, and the Quantum Physics Commons

\section{Recommended Citation}

Heelan, Patrick A., "The Intentionality Structure of Complementarity" (1965). Research Resources. 17. https://fordham.bepress.com/phil_research/17

This Article is brought to you for free and open access by the Hermeneutic and Phenomenological Philosophies of Science at DigitalResearch@Fordham. It has been accepted for inclusion in Research Resources by an authorized administrator of DigitalResearch@Fordham. For more information, please contact considine@fordham.edu. 
CHAPTER THREE

\title{
THE INTENTIONALITY STRUCTURE OF COMPLEMENTARITY
}

\author{
SECTION I: BOHR AND COMPLEMENTARITY
}

\section{The Copenhagen Spirit}

Heisenberg's opposition to wave mechanics did not last long. Influenced by Bohr, he came to accept its elegant mathematical methods - though not Schrödinger's interpretation of them - and also the more concrete manner of presentation afforded by Bohr's Principle of Complementarity. In the preface to the lectures he gave at the University of Chicago in the spring of 1929 and published under the title The Physical Principles of the Quantum Theory, he speaks of the "conclusive studies of Bohr in 1927" on the nature of the quantum theory, and identifies himself completely with the Kopenhagener Geist der Quantentheorie founded, he says, upon the "complete equivalence of corpuscular and wave concepts". "What, was born in Copenhagen in 1927", he wrote, "was not only an unambiguous prescription for the interpretation of experiments, but also a language in which one spoke about Nature on the atomic scale, and in so far a part of philosophy" 1. Heisenberg's acceptance of wave mechanics as an intrinsic part of the quantum theory was understandable; but his capitulation to the philosophy of complementarity was, in our opinion, unfortunate; for it led him away from the true sense of his original insight 2.

1 W. Heisenberg, "The Development of the Interpretation of the Quantum Theory" in Niels Bohr and the Development of Physics, ed. by W. Pauli (Oxford: Pergamon Press, 1955), p. 16.

2 It is often said and generally believed that the quantum theory owes its origin and inspiration to the spirit of complementarity. On this point, P. K. Feyerabend writes not altogether unjustly: "The full quantum theory we owe to a metaphysics diametrically opposed to the philosophical point of view of Niels Bohr and his disciples, viz., to that of Schrödinger. This is quite an important historical fact as the adherents of the Copenhagen picture very often criticize the metaphysics of Bohm and Vigier by pointing out that no physical theory has yet been developed on the basis... They forget that the Copenhagen way of thinking has not produced a theory either. What it has produced is the proper interpretation of Schrödinger's wave mechanics after this theory has been introduced. For it turned out that Schrödinger's 


\section{Complementarity}

The Principle of Complementarity is not a physical principle like the the Indeterminacy Principle, nor is it a heuristic principle in physics like the Correspondence Principle; but it is a philosophical (or epistemological) principle which attempts to explain how we know the atomic domain and how the inherent limitations of our knowing powers impede our conception and expression of the intrinsic nature of microphysical events 1 .

In the seven paragraphs immediately following we give our summary of the philosophy of complementarity with some comments on the import of the doctrines involved.

(i) The aim of atomicphysics is to put order into an increasing range of our experiences with a view to predicting patterns in these experiences 2. The Galilean and Newtonian aim of trying to find out the truth about the heavens and the earth is to be abandoned for the reason alleged by Bohr that the interior resources we have for investigating the atomic domain are too modest to allow us ever to reach such a truth.

(ii) The resources of our knowing powers are limited by the "forms of perception" 3 which allow us to speak and to describe only (what we have called) bodies in the strict sense, and to conceive only inductive generalisations of bodily phenomena. The two kinds of bodies are particles which are localisable and fields which are non-localisable,

wave mechanics was just that complete rational generalisation of the classical theory that Bohr, Heisenberg and their collaborators had been looking for and parts, of which they had already succeeded in developing", in "Problems in Microphysics", Frontiers of Science and Philosophy (London: Allen and Unwin, 1964), pp. 264-266.

1 References will be made chiefly to the following works of N. Bohr: Atomic Theory and the Description 01 Nature (Cambridge: 1961), which is a collection of four of Bohr's most celebrated articles on the interpretation of quantum mechanics published originally between 1925 and 1929, with an introductory survey and commentary by the author written in 1934; "Can Quantum Mechanical Description of Physical Reality be Complete?", Phys. Rev., XLVIII (1936), 696; "On the Notions of Causality and Complementarity", Dialectica, 7/8 (1948), pp. 312-319; "Discussions with Einstein on Epistemological Problems in Atomic Theory", in Albert Einstein: Philosopher-Scientist, pp. 199-242. For a detailed study of Bohr's philosophy of complementarity by a modern philosopher-physicist, of., P. K. Feyerabend, "Complementarity", in Proc. Aristot. Soc., Suppl. Vol., XxxII (1958), pp. 75-104. It should be noted that the philosophical doctrine called Complementarity has undergone considerable evolution, notably on the part of Heisenberg and in a sense away from the predominantly empiricist spirit of the early phase. For the essentials of what might be called the irreducible physical principles of complementarity, see Concept of the Positron, chaps. VI-VII, by N. R. Hanson. It is in the sense of Hanson that most physicists belong to the Copenhagen School. However, there is a big difference between Hanson's summary and the unabridged philosophies of Bohr and Heisenberg.

2 Bohr, Atomic Theory etc., pp. I, 12, 16-17, 55, 69, 77, 87.

3 Ibid., pp. 1,5,15-19,22,90-93,96,103, III; Albert Einstein, etc., pp. 209; Dialectica, p. 313; Pkys. Rev., loco cit., p. 702. 
although a field may give rise to a wave packet of virtually finite dimensions. Thus, the forms of perception "idealise" every experience and notably those of atomic phenomena by submitting them to a synthesis in which the velocity of light is represented as infinitely large, and Planck's constant is represented as vanishingly small!. In other words, every experience of atomic phenomena is "idealised" either as a particle or as a wave. This is what is meant by wave-particle duality. This idealisation, however, is due to our forms of perception and is neither a coherent objective picture of the atomic event taken as a phenomenon nor does it give us true knowledge of the atomic event as a reality 2. Bohr was a realist in the empiricist sense; that is, he held that reality, if it is to be known truly, can be known only in and through a stable and coherent phenomenal object.,Since no coherent phenomenal object can be formed of an atonlic event, he considered that the atomic event cannot be known as a reality, that is, as it is in itself.

(iii) The concepts of quantum mechanics are defined in terms of the concepts of classical physics. These are just refinements of the concepts of everyday life and refer only to bodies in the strict sense 3.

(iv) Our experience of atomic phenomena occurs within acts of observation. These involve a union between the knowing subject and the known object in which no sharp distinction can be made between them. Moreover, the observing subject disturbs its object in the act of observing it: the disturbance of macroscopic objects is very small, but in the atomic domain the disturbance is considerable and, moreover, inescapable, since the subject and the object must share between them at least one indivisible quantum of energy 4. Hence, our (private) subjectivity enters essentially and inevitably into our experience of atomic phenomena. This theory of observation is founded upon what we shall call the perturbation theory ot measurement. This completes the two aspects under which our knowledge is non-objective. (The first was mentioned above in (ii).

(v), A consequence of this is that (bodily or phenomenal) causality does not hold for atomic phenomena. Hence the statistical laws of the atomic domain are irreducible 5. Bodily (or phenomenal) causality, it

1 Bohr, Atomic Theory etc., PP 5, 16-17, 22, 66, 116.

2 Ibid., pp. I, 5, 96-97, 116.

3 Ibid., pp. I, 5, 8, 17, 53.

4 Ibid., pp. I, 4-5, I0-II, 22, 53-54, 67, 93-96, II4, 119; Albert Einstein etc., pp. 224; Dial., loco cit., pp. 313, 317.

5 Bohr, Atomic Theory etc., pp. 4, 5, 13, 57-61, II7. 
will be renlembered, is the law-like association in a continuous temporal sequence of spatially organised bodies (or phenomena).

(vi) The function of mathematical theory is not merely to be an «indispensable tool for describing quantitative relationships", like Heisenberg's matrix mechanics, it is also "an essential means for the elucidation of the general qualitative points of view" 1, that is, it can also be used to describe the two complementary viewpoints represented by the wave and particle pictures.

(vii) The "reduction (or contraction) of the wave packet" which is the name given to what takes place in an act of observation, is partly a physical effect since it results from the physical union of subject and object. It is partly a psychological effect in so far as the subject translates this uniquely into a psychological act of observation: this translation takes place according to the Principle of Psycho-physical Parallelism 2. And it is partly a logical effect, since, as Feyerabend points out 3, the subject switches suddenly from a wave-type or field-type description to a particle-type description.

The way of complementarity then consists in "liberalising" our classical concepts 4 . This means knowing when to use a particle representation and when to use a field or wave-like representation to order our experiences. Every statement of fact in the quantum theory is necessarily a statement in terms of classical concepts, that is, in terms of the concepts of classical particle physics and in terms of the concepts of classical field physics. As Reichenbach has well said of the duality of wave and particle: "The and is not in the language of physics, but in the metalanguage, that is in a language which speaks about the language of physics... It does not refer to the physical object but to possible descriptions of the physical object and thus falls into the realm of the philosopher" 5 .

The kind of physical theory based upon complementarity is called by Bohr a «rational generalization of classical physics" 6. The Correspondence Principle is, accordingly, a kind of transcendental deduction from the Principle of Complementarity 7.

1 Ibid., p. 8.

2 Ibid., pp. 24, II8; ct., intra, chap. IV.

3 Feyerabend, loco cit., p. 95.

4 Bohr, Atomic Theory etc., pp. 3, 5, IB, 63.

5 Hans Reichenbach, Rise of Scientific Philosophy, (Berkeley: Univ. of Cal. Press, 1962), pp. $175-176$.

6 Bohr, Atomic Theory etc., pp. 4, 19,70,87,92, II0; Dial., lococit., p. 316; Albert Einstein etc., pp. 210, 239.

7 Bohr, Atomic Theory etc., pp. 37, 70, 80̂, IIO; ct., infra, chap. VI. 


\section{SECTION II: HEISENBERG AND COMPLEMENTARITY}

\section{Heisenberg and Complementarity}

That his acceptance of complementarity made Heisenberg vaguely uneasy at the beginning is suggested by the account he wrote in 1960 , and to which we have frequently referred 1 . In this he goes out of his way to note that he preferred a different approach to quantum mechanics from Bohr's. Not only was there the question of discontinuities in nature, but their viewpoints and casts of mind were different. Bohr's approach to physics was empirical, and moved from the phenomena to theory, which he considered to be a movement from a lesser generalisation to a greater one; Heisenberg on the other hand took a more intellectualistic approach to physics, moving from the potentialities of theory to the testing of these in phenomena 2. Bohr could be classified as empiricist and positivist in his metaphysics; while Heisenberg on the other hand was, and became increasingly with the lapse of time, an idealist. Both, however, called themselves realists; but for different reasons; Bohr because for him the reality of everyday life (and classical physics) was the really real which he understood in an empiricist sense, while Heisenberg called himself a realist because he never lost sight of the transcendent object which, though unknowable, was the noumenal correlate of the phenomena. This difference in viewpoint showed up in their different interpretations of the Indeterminacy Relations: as Landé put it; Bohr affirmed the Unschiirte des Seins, while Heisenberg asserted merely the Unbestimmtheit der Voraussage 3. It was only because they could agree in their interpretation of the phenomenal plane that Heisenberg could with sincerity accept the premises of complementarity. However, while complementarity constituted the whole horizon of Bohr's philosophy, it was really only an element -let us say, a premise - for the gradual elaboration of Heisenberg's metaphysics. Instartingpoint, in problematic and in systematic conclusions, Heisenberg played Kant to Bohr's Hume.

\section{Wave-particle Duality in Heisenberg}

In the Physical Principles ot the Quantum Theory, Heisenberg paid tribute to the "conclusive studies of Bohr" and dedicated his work to

1 Heisenberg, Erinnerungen usw.

2 Heisenberg, Niels Bohr etc., pp. 12-29, especially p. I5.

3 A. Lande, "Dualismus, Wissenschaft und Hypothese", in Werner Heisenberg und die Physik unserer Zeit, p. 124. 
the "diffusion of the Kopenhagener Geist der Quantentheorie... which has directed the entire development of modern physics 1.

All our concepts, he says, are attempts to "clarify and synthesise results" 2 which are described in classical concepts, i.e., in the "language of everyday life" 3 . These concepts are idealisations in which "both the gravitational constant and the reciprocal of the velocity of light may be regarded as negligibly small" 4. They refer exclusively to things of which we can form a picture in the imagination 5. All our concepts, except mathematical concepts, are formed by inductive generalisations of experience, but the limits of their applicability are to be determined by reference to experience 6. Particle and wave properties of both light and matter, which are intimately linked in experiment, are too different to be simultaneous properties of the same thing. Hence, we are led to form "two mental pictures-one of a particle and the other of waves-both incomplete". They are complementary and mutually exclusive aspects of atomic phenomena. Each has a limited domain of applicability and neither must be "pushed too far" 7 . When we speak, we refer to one or other of these pictures, but neither is a true visualisation of the atomic event. Atomic phenomena cannot be explained as relations between objects existing in space and time 8. Consequently, our knowledge of events is an inextricable mixture of subjective and objective elements 9 .

A traditional requirement of science has been a sharp division between subject and object, i.e. between observer and observed 10. This is possible in classical physics where the interaction between them is negligible 11. In the atomic domain, however, the interaction can cause ('uncontrollable and large changes in the system observed" 12. The union between the observer and the observed is such that it is impossible to determine what part of a system belongs to one and what part to the other 13. This leads to a certain inescapable indetermincay in our knowledge of the simultaneous values of certain quantities,

1 Heisenberg's Preface to Physical Principles of the Quantum Theory.

2 Heisenberg, Physical Principles etc., p. 1.

3 Ibid., pp. 1-3.

4 Ibid., p. 2.

5 Ibid., p. 11.

6 Ibid., pp. 1, 11.

7 Ibid., pp. 10, 64, chaps. II and III.

8 Ibid., pp. 63-64.

9 Ibid., p. 65 and passim.

10 Ibid., p. 2.

11 Ibid., p. 3.

12 Ibid., pp. 3, 64, 20-46.

13 Ibid., pp. 58, 64, 67. 
which is expressed in the Indeterminacy Relations 1. This indeterminateness is attached to our knowledge of each individual microscopic object 2. Our knowledge then of this class of objects is limited to irreducible statistical distributions and correlations 3.

\section{Causality in Heisenberg}

The Principle of Causality expressed in the form "Natural phenomena obey exact laws" is to be renounced. Causality, he says, can be defined only for an isolated system 4. No set of atomic systems, in so far as they are the objects of observation-acts, obeys the law of causality, since the act of observation disturbs them, and this disturbance precludes the exact geometrical description of each which is a necessary condition for causality 5 . The disturbance is due to the sharing of ultimate and indivisible entities (viz., quanta of energy) 6 . The influence of the measuring device which brings about a discontinuous change in the system "is treated in a different manner from the interaction of the various parts of the system"; these on the other hand enter determinately and causally into the description 7 . The discontinuance change produced by an act of measurement is called the "reduction of the wave packet". It is a physical effect since it effects a change in the object 8 . It is a psychological effect since it results in a discontinuous change in our knowledge 9. It is a logical effect since it results in a change of the mathematical representation of the physical process from a wave to a particle picture $\mathbf{1 0}$.

Heisenberg has reaffirmed all the propositions of this section and of the preceding one many times since 1929 , and he has given more detailed and explicit treatment of some of the key ideas. For example in 1931, he gave a conference to a group of physicists and philosophers in which he described the crisis produced in the concept of causality and in the Law of Causality by quantum mechanics. Most of the members of the

1 Ibid., pp. 13-46.

2 Ibid., pp. 2,2 52.

3 Ibid., pp. 33-34, and passim.

4 Ibid., pp. 62 -63. Note that Heisenberg often uses the term "causality" in a wider sense than Bohr, as referring to any determinate connection parametrised by time between entities, whether these be mathematical, phenomenal or bodily entities.

5 Ibid., pp. 58, 63 and passim.

6 Ibid., p. 63.

7 Ibid., p. 58. Note that this implies a perturbation theory of measurement, viz., that the measured object is disturbed by the measurement and its true properties thereby obscured by it.

s Ibid., p. 39 and passim.

9 Ibid., p. 36 and passim.

10 Ibid., p. 36. 
group, like J. von Neumann, P. Frank and H. Reichenbach, belonged to the Vienna Circle. The conference was published in Erkenntnis under the title "Kausalgesetz und Quantentheorie" 1.

In this conference, Heisenberg examines five different formulations of the Law of Causality and finds them either tautologous, not falsifiable or inhaltsleer. A formulation is tautologous if it merely analyses the sense of the words without affirming the existence of a referent for this sense. A non-falsifiable proposition is one whose referents, if there are such, are inaccessible to human investigation. A concept is inhaltsleer if it has no referent, that is, if there is nothing in fact in which it is verified. A law is inhaltsleer if it has no observable consequences 2. The sense of inhaltsleer corresponds to the positivist notion of ohne Bedeutung (often translated by meaningless).

Heisenberg finds that the classical Law of Causality (viz., that characteristic of classical physics) is inhaltsleer (presumably in atomic physics only), since the conditions of applicability of its concepts and hence of the law are never fulfilled. The classical law of causality can be formulated: "In an isolated system, if the present state of the system is known in all its particulars, then the future state of the system can be calculated". Heisenberg points out that an isolated system is an unobserved system, since the act of observation would result in a fusion of the system with a knowing subject, thus removing its isolation as well as disturbing its original state. Moreover, since the Uncertainty Principle does not permit exact knowledge of all the variables of the initial state of the system, the conditions for the fulfilment of the classical physical law of causality are never in fact fulfilled. He considers the substitution of "ray in Hilbert space" (or "wave function") for the "present state of the system" and notes that the Schrödinger equation describes deterministically the time-change of this ray (or wave function). This species of causality, he says, is not real causality since the space of the wave function is configuration space (i.e., an abstract theoretical3n-dimensional space where $\mathrm{n}$ is the number of particles) and not the space of observable events 3 . Ob-

1 Heisenberg, Erkenntnis, loco cit.

2 Ibid., p. 173.

3 Heisenberg distinguishes between two kinds of waves: the "configuration space wave" which is also the wave function of a many particle system, and the "probability wave" or the "wave packet". The former is an ideal mathematical construction in a space of $3 \mathrm{n}$ dimensions (ct., Physical Principles etc., Preface; Philosophic Problems etc., p. 15). The latter is the three-dimensional matter wave associated with the complementary particle picture. Within the perspective of complementarity, the "probability wave" has as much "reality" as the particle, i.e., both are equivalent ways of speaking about the same physical object; ct., Niels Bohr etc., p. 24; Physical Principles etc., p. 13. 
servable events are not connected causally, but - except in the case of commuting operators - only statistically. Nor is there any escape from indeterminism by including the observer (i.e. the eyes and the other senses of the scientist as well as his instruments) within the system in order to construct a more inclusive but isolated system of which the observer is now a part; for this just leads to a more comprehensivewave function which has in turn to be reduced to one of the possibilities it contains by some super-act of observation. There is no way, he concludes, of setting up or predicting a determinate chain of observable events in quantum mechanics.

Of the Kantian expression of the Law of Causality, viz., that there is a unique antecedent-consequent connection between objectifiable events, Heisenberg concludes that it too is inhaltsleer, since atomic events - because of the Indeterminacy Principle - cannot be objectified in the Kantian sense. He compares the status of the Law of Causality with the principles of Euclidean geometry. Both are synthetic a priori principles according to Kant. Since Einstein's relativity theory has shown that a gravitational field is characterised by a nonEuclidean geometry, we are justified in saying only that space is Euclidean, if the conditions for the fulfilment Euclidean geometry are fulfilled. This last is an a posteriori condition and might not in fact be fulfilled, or might be fulfilled only in certain cases. Similarly, with the Kantian Law of Causality, the conditions for its application might not be fulfilled and are not in fact fulfilled in atomic physics. Since Kant has shown that the Law of Causality is a necessary condition for objective science, he concludes that quantum mechanics is not objective. The objectivity in question is that attributed by Kant to all empirical scientific objects. This is (what we have called) phenomenal objectivity. The non-objectivity of a quantum system is shown by the fact that it is known only to the extent that it interacts with an observer-subject. Heisenberg concludes from this that modern physics is not concerned with the essence and structure of the atom but with observable events. The emphasis is thus placed upon the measurement process. He adds as an afterthought that, although the Law of Causality is no longer universal, causality holds between successive repetitions of a measurement since these give the same (or neighbouring) values for the measured quantity.

During the course of the subsequent discussion, he asked whether, in the reduction of the wave packet, the selection of one observed value out of the many possible values is to be explained by the 
physiology of the human observer. He replies that a photographic plate can play the part of an observer equally well: "aber das ist noch kein Zusammenhang zwischen der Physik und psychologischen Fragen" 1. It is doubtful whether, given the later development of measurement theory along the lines suggested by complementarity, he would give the same answer to-day.

In a reply to von Neumann, he puts on record his opposition to any way of speaking which would identify the wave function with the Ding an sich. In the later development of his thought, he would affirm a Ding an sich represented by the wave function, to which he would give the name potentia or objektive Tendenz 2.

\section{Heisenberg's View of Physics}

Heisenberg's Principielle Fragen der Modernen Physik (1936) and his Wandlungen in den Grundlagen der Naturwissenschaft (8th edition, 1949) which are collections of occasional lectures delivered between 1932 and 1948, restate without change or development the propositions we have enunciated above 3. In these lectures, Heisenberg shows himself interested in the historical and dialectical development of the concept of nature from the Middle Ages up to the present day 4. $\mathrm{He}$ sees himself justly in the line of those who helped to change man's view of nature and, reflexively, his view of himself vis-à-vis nature.

We have pointed out that Heisenberg's approach to physics was from the starting point of theory, Le., from the free creative uninhibited search for mathematical theories a priori to external empirical experience. These played the part of Kant's pure science of nature, not to the extent that they laid down absolute and necessary conditions of possibility which every scientific object obeys, but to the extent that they served to define conditions of possibility for possible scientific objects. A theory which is inhaltsleer is distinguished from one which has observable consequences by having recourse to empirical tests 5 . He held that neither the forms of thought, nor experience itself imposed on us a unique a priori or pure science of nature. The representative value and the limits of applicability of each theory were to be de-

1 Ibid., p. 184.

2 See intra, chap. VIII, pp. 150-152.

3 These two volumes have been translated into English and appear together under the title: Philosophic Problems of Nuclear Physics (London: Faber and Faber, 1952).

4 Ibid., pp. 12-13; also W. Heisenberg, "Der Begriff 'Abgeschlossene Theorie' in der modernen Naturwissenschaft", Dialectica, II, 7/8 (1948), pp. 331-336.

5 Heisenberg, Physical Principles etc., p. 15. 
termined a posteriori by experimental investigation 1 . In this respect, he abandoned both the rationalisitc intentionality-structure of classical physics, and the rigid set of synthetic a priori principles which Kant alleged were necessarily operative in the construction of every scientific object. A theory verified within a limited domain was given the name "abgeschlossene Theorie" (a Closed or Complete Theory) 2. Heisenberg gave four examples: (a) Newtonian Mechanics, (b) Maxwell's electromagnetic theory, (c) heat and statistical mechanics, and (d) the quantum theory with chemistry annexed. We shall return to the notion of a Closed (or Complete) Theory later on.

In a paper entitled Wahrscheinlichkeitsaussagen in der Quantentheorie der Wellenfelder (1938) 3, Heisenberg again reiterates his view that the act of measurement disturbs the object and thereby sets limits to our knowledge; that such knowledge as we are capable of, is penetrated with an inescapable (private) subjectivity; and that, consequent on the inter-penetration of subject and object in physics, the laws of atomic physics are irreducibly probabilistic.

This account brings us approximately up to the year 1950. As the subsequent development of his philosophy concerned principally the ontology of nature, we shall postpone the consideration of his later works of philosophical interest to Part II. We shall summarise below some of the principles and conclusions of Heisenberg's philosophy with regard to scientific method and the structure of knowledge. Our criticism of these will occupy the next three chapters.

SECTION III:

THE INTENTIONALITY STRUCTURE OF COMPLEMENTARITY

Complementarity in its original form contains two key ideas on scientific method and three basic propositions in philosophy. The two sets of ideas are intimately related and constitute a logical whole which we might call the intentionality-structure of complementarity in its early phase.

The key propositions on scientific method are the following: (r) The definition of variables can only be made with the aid of classical physical concepts. These are identical- except for refinements

1 Heisenberg, Philosophic Problems etc., p. 23; and Zeit.f. Physik, XliII (1927), p. 172.

2 Heisenberg, Philosophic Problems etc., p. 24; and Dial., loco cit., pp. 331-336.

3 W. Heisenberg, "Wahrscheinlichkeitsaussagen in der Quantentheorie der Wellenfelder", Actualités scientifiques et industrielles, No. 734 (Paris: Hermann, 1938). 
with the concepts of everyday life; (2) The act of measurement perturbs the object. Its objective state ("objective" that is, "not affected by the subjectivity of purely private experience") cannot be known - whether as an object of empirical science (a phenomenal object) or as a reality (an object in the strict or formal sense). The Indeterminacy Principle expresses the degree of this perturbation, and, at the same time, traces the limits of our power of knowing physical objects. We call this the perturbation theory of measurement.

Three basic philosophical propositions are linked with these 1. (I) It is impossible for us to know atomic events in their transcendent noumenal reality or in an objectifiable phenomenal reality, since the resources of our knowing powers are limited to manipulating and synthesising phenomenal representations. Two kinds of phenomenal representations are available to us, wave representations and particle representations, and out of these our knowledge of atomic systems has to be constructed. The resulting construction lacks both formal (or strict) objectivity and empirical objectivity. (2) Causality (viz., the strict temporal antecedent-consequent link between bodies or phenomena) fails in atomic physics and for this reason atomic systems are not empirically objectifiable, and for the same reason the statistical laws of quantum physics are irreducible. (3) The only knowledge which has a right to be called objective knowledge of physical reality is an observation-event: this is the perception of an everyday event occurring in (three-demensional) space and ordinary time. With regard to atomic systems, our knowledge is not objective - even in the sense of public objectivity. We know such systems only in an observationevent which is the indissoluble union of observer (subject) and observed (object) and to such knowledge is attached an irreducible element of the subjectivity of private experience. Quantum mechanics may be said to have a public instrumental value or public objectivity only for the purpose of (statistical) prediction or technical use. As far as individual systems are concerned, our knowledge falls short even of public objectivity.

\section{Summary}

The return to the concrete and empirical implied in Heisenberg's insight on the importance of observables in physics was not, however,

1 The philosophical propositions outlined below belong to a phase which lasted in Heisenberg's case up to approximately 1950. After that, the predominantly rationalist and Kantian bent of his mind separated him more and more from Bohr and the empiricist wing of the Copenhagen School. A comparison will be made in Part II between Heisenberg's early and late philosophy. 
in Heisenberg's case, accompanied by a thorough re-thinking of the rationalist presuppositions of classical physics. The effect on Bohr, however, was to lead him to a complete rejection of rationalism and to the adoption of the contrary extreme, empiricism. The profound though largely implicit - cause of the disagreement between Bohr and Heisenberg as to the correct interpretation of quantum mechanics, was resolved in the sumnler of 1927 , by the common acceptance of the philosophy of complementarity. This was based upon the acceptance of wave mechanics - though not of Schrödinger's interpretation of it as an equally valid part of the quantum theory with matrix mechanics. A corollary of this was agreement about the complete equivalence of wave and particle representations of quantum phenomena. The latter was called wave-particle dualism or the Principle of Complementarity. The common acceptance of complementarity resulted in agreement as to the language in which quantum phenomena were to be described. In this chapter, we stated the essential propositions of the philosophy of complementarity concerning the nature and limits of human knowing, scientific method and the ontology of nature. 